\title{
Quality of life: Transfusion dependent thalassemia vs non-transfusion dependent thalassemia
}

\author{
Mehran Karimi, Nader Cohan \\ Hematology Research Center, Shiraz University of Medical Sciences, Shiraz, Iran
}

\section{Background}

Although the improvements in the treatment and management of thalassemia patients in new years lead to the improved survival and quality of life (QOL) in this group of patients, QOL is still is an important dimension of care in thalassemic patients (1). WHO defines QOL as "an individual's perception of their position in life in the context of the culture and value systems in which they live and in relation to their goals, expectations, standards, and concerns" (2). Thalassemia is a chronic disease needs life-long care with multiple physical, mental and social complications that affect QOL in patients. The most important factors which affect QOL in thalassemia are: effects of the disease on family, skeletal and face changes, frequent blood transfusion and drug infusion, sexual impairment and infertility, heart and liver disease as well as endocrine disorders, anxiety and low life expectancy (3).

\section{Discussion}

Although thalassemia major (TM) is a life-long transfusion dependent, thalassemia intermedia (TI) is a milder form of the disease and may be non-transfusion dependent. QOL was evaluated frequently in TM (4-8) but its situation in TI and compare to TM is not well evaluated. In the first report by pakbaz et al., 29 transfusion dependent TM and 19 transfusion independent TI patients were evaluated for QOL by Dartmouth Care Cooperative Chart System (COOP) questionnaire in children (mean age of 12 years old for TI and 17 years old for TM). Overall they found that QOL is more impaired in non-transfusion dependent patients compared to transfusion dependent (9). Also, Musallam et al., compared QOL in adult patients with non-transfusion dependent (32 TI, median age 24 years old) and transfusion dependent (48 TM, median age 23 years old) thalassemia patients by the RAND SF36 survey (10). In agreement with the previous study, they also found overall health-related QOL impaired in TI patients compared to TM patients. In the study by Musallam et al., both physical and mental health score was significantly lower in TI patients

Correspondence: Mehran Karimi, Professor of Pediatric HematologyOncology, Hematology Research Center, Shiraz University of Medical Sciences, Nemazee Hospital, Shiraz, Iran.

Tel/Fax: +987136473239 .

E-mail: mkarimi820@gmail.com

This work is licensed under a Creative Commons Attribution 4.0 License (by-nc 4.0).

CCopyright M. Karimi and N. Cohan, 2018

Licensee PAGEPress, Italy

Thalassemia Reports 2018; 8:7489

doi:10.4081/thal.2018.7489 compared to TM patients (10). The limitation of these two studies was a low number of the study population. In a recent study, we also evaluated the QOL in a larger TI study group (118 TI, $26.5 \pm 6.5$ years old) and compared with $101 \mathrm{TM}(19.5 \pm 4.4$ years old) patients by SF36 questionnaire (11). Physical functioning was the best QOL score in TI patients in this study. In compare between TI and TM patients the total score was similar $(66.5 \pm 15.4$ for TI and $67.8 \pm 16.1$ ) and based on subscales score, only physical functioning showed a better condition in TM compared to TI $(86.9 \pm 12.9$ for $\mathrm{TM}$ and $76.8 \pm 26.6$ for $\mathrm{TI}, \mathrm{P}<0.0001)$ (11). Although in this study unlike the previous studies health-related QOL in TI was not impaired compared to TM it was not also better as expectations. An Intercontinental Collaborative Study was conducted on 38 non transfusion dependent thalassemia (NTDT) and 97 transfusion dependent thalassemia (TDT) patients. All patients were over 18 years old from Canada, Iran and Lebanon. This study showed QOL of NTDT is better than TDT patients at younger age, but, while NTDT patients may not require regular transfusions based on conventional criteria, they may experience significant reduction in QOL especially at older ages. It seems long-term NTDT complications may contribute to find QOL is not better than TM at older age (12).

\section{Conclusions}

Thalassemia leading to reduce health related-QOL in affecting patients including physical, mental and social capabilities. As expected based on the studies, the QOL in non-transfusion dependent thalassemia is not better that transfusion dependent patients. Improving QOL and life expectancy in a chronic disease like thalassemia is the most important management approach in these groups of patients. Thalassemia centers and clinics should evaluate QOL in patients especially in non-transfusion dependent patients to determine best management modalities and improve QOL. Early and precise diagnosis, routine monitoring, parents and patient's education, financial support as well as enhancement in mental and psychosocial conditions are essential for improving QOL in thalassemic patients.

\section{References}

1. Gollo G, Savioli G, Balocco M, Venturino C, Boeri E, Costantini M, Forni GL. Changes in the quality of life of people with thalassemia major between 2001 and 2009. Patient Prefer Adherence. 2013;7:231-6.

2. http://www.who.int/healthinfo/survey/whoqol-qualityoflife/en/

3. Telfer P, Constantinidou G, Andreou P, Christou S, Modell B, Angastiniotis M. Quality of life in thalassemia. Ann N Y Acad Sci. 2005; 1054:273-82.

4. Maheri A, Sadeghi R, Shojaeizadeh D, Tol A, Yaseri M, Ebrahimi M. Associations between a health-promoting lifestyle and quality of life among adults with beta-thalassemia major. Epidemiol Health. 2016;38:e2016050. 
5. Abu Samra O, Auda W, Kamhawy H, Al-Tonbary Y. Impact of educational programme regarding chelation therapy on the quality of life for B-thalassemia major children. Hematology. 2015;20(5):297-303.

6. Siddiqui SH, Ishtiaq R, Sajid F, Sajid R. Quality of life in patients with thalassemia major in a developing country. J Coll Physicians Surg Pak. 2014;24(7):477-80.

7. Tuysuz G, Tayfun F. Health-related Quality of Life and its Predictors Among Transfusion-dependent Thalassemia Patients. J Pediatr Hematol Oncol. 2017;39(5):332-336.

8. Dhirar N, Khandekar J, Bachani D, Mahto D. Thalassemia Major: how do we improve quality of life? Springerplus. 2016;5(1):1895.

9. Pakbaz Z, Treadwell M, Yamashita R, Quirolo K, Foote D, Quill L, et al. Quality of life in patients with thalassemia inter- media compared to thalassemia major. Ann N Y Acad Sci. 2005; 1054:457-61.

10. Musallam KM1, Khoury B, Abi-Habib R, Bazzi L, Succar J, Halawi R, et al. Health-related quality of life in adults with transfusion-independent thalassaemia intermedia compared to regularly transfused thalassaemia major: new insights. Eur J Haematol. 2011 Jul;87(1):73-9.

11. Haghpanah S, Vahdati S, Karimi M. Comparison of Quality of Life in Patients with $\beta$-Thalassemia Intermedia and $\beta$ Thalassemia Major in Southern Iran. Hemoglobin. 2017; in press.

12. Amid A, Leroux R, Merelles-Pulcini M, Yassobi S, Antoine N Saliba, Ward R. et al. Factors Impacting Quality of Life in Thalassemia Patients; Results from the Intercontinental Collaborative Study. 58 $8^{\text {th }}$ annual ASH meeting. Blood Journal abstract.2016 Dec. Vol. 128, Issue 22. 